This is the submitted version of the following article:

Fabri-Faja N., Calvo-Lozano O., Dey P., Terborg R.A., Estevez M.-C., Belushkin A., Yesilköy F., Duempelmann L., Altug H., Pruneri V., Lechuga L. M.. Early sepsis diagnosis via protein and miRNA biomarkers using a novel point-of-care photonic biosensor. Analytica Chimica Acta, (2019). . : - . 10.1016/j.aca.2019.05.038,

which has been published in final form at https://dx.doi.org/10.1016/j.aca.2019.05.038 () https://dx.doi.org/10.1016/j.aca.2019.05.038. This manuscript version is made available under the CC-BY-NC-ND 4.0 license http://creativecommons.org/licenses/by-nc-nd/4.0/ 


\section{Early Sepsis Diagnosis via Protein and miRNA Biomarkers using a novel Point-of-Care Photonic Biosensor}

Nuria Fabri-Faja ${ }^{1}$, Olalla Calvo-Lozano ${ }^{1}$, Priyanka Dey ${ }^{1}$, Roland A. Terborg ${ }^{2}$, M.-

Carmen Estévez ${ }^{1,}$, Alexander Belushkin ${ }^{3}$, Filiz Yesilköy ${ }^{3}$, Luc Duempelmann², Hatice Altug $^{3}$, Valerio Pruneri2,4, Laura M. Lechuga ${ }^{1}$

1) Nanobiosensors and Bioanalytical Applications Group, Catalan Institute of Nanoscience and Nanotechnology (ICN2), CSIC, CIBER-BBN and BIST, Campus UAB, 08193 Bellaterra

(Barcelona), Spain

2) ICFO-Institut de Ciències Fotòniques, The Barcelona Institute of Science and Technology, 08860 Castelldefels (Barcelona), Spain

3) Institute of Bioengineering, École Polytechnique Fédérale de Lausanne (EPFL), Lausanne 1015, Switzerland

4) ICREA—Institució Catalana de Recerca i Estudis Avançats, 08010 Barcelona, Spain 


\section{ABSTRACT}

Sepsis is a condition characterized by a severe stage of blood-infection often leading to tissue damage, organ failure and finally death. Fast diagnosis and identification of the sepsis stage (sepsis, severe sepsis or septic shock) is critical for the patient's evolution and could help in defining the most adequate treatment in order to reduce its mortality. The combined detection of several biomarkers in a timely, specific and simultaneous way could ensure a more accurate diagnosis. We have designed a new optical pointof-care (POC) device based on a phase-sensitive interferometric biosensor with a label-free microarray configuration for potential high-throughput evaluation of specific sepsis biomarkers. The sensor chip, which relies on the use of metallic nanostructures, provides versatility in terms of biofunctionalization, allowing the efficient immobilization of different kind of receptors such as antibodies or oligonucleotides. We have focused on two structurally different types of biomarkers: proteins, including C-reactive protein (CRP) and Interleukin 6 (IL6) and miRNAs, using miRNA-16 as example. Limits of Detection (LoD) of $18 \mu \mathrm{g} \mathrm{mL}^{-1}, 88 \mu \mathrm{g} \mathrm{mL}^{-1}$ and $1 \mu \mathrm{M}\left(6 \mu \mathrm{g} \mathrm{mL}^{-1}\right)$ have been respectively obtained for CRP, IL6 and miRNA-16 in individual assays, with high accuracy and reproducibility. The multiplexing capabilities have also been assessed with the simultaneous analysis of both protein biomarkers.

Keywords: Point-of-care; label-free multiplexed biosensing; nanoplasmonics; sepsis; protein and miRNA biomarkers; microarray 


\section{Introduction}

Sepsis is a condition expressed as a critical whole-body inflammatory response due to an infection. The first stage of sepsis involves a systemic inflammatory response (SIRS), followed by a severe sepsis which is characterized by organ dysfunction (liver, kidney, lung and heart). Further deterioration results in a sepsis shock that could cause hypotension and, ultimately, death [1]. Currently, sepsis is the main cause of death in Intensive Care Units (ICU) and its incidence is increasing worldwide with a mortality rate between 40 to $50 \%$ in developed countries [1-4]. The mortality rate increases with delayed diagnosis or with inappropriate antibiotic therapy, which implies, besides the human losses, in a high economic cost for the healthcare systems [4]. A well-timed and precise diagnosis of sepsis is really essential for the on-time selection of the most appropriate therapy.

Diagnosis of sepsis is not straightforward. There has been an extensive clinical research focused on identifying a reliable panel of biomarkers associated to sepsis. Currently, there are more than 170 different biomarkers that could be useful for either its diagnosis or prognosis [4]. Some of them are already used at clinical level for diagnosis and treatment monitoring. Different pro-inflammatory cytokines (TNF, IL-1 $\beta$ and IL6) as well as C-reactive protein (CRP) and procalcitonin (PCT) have been proposed, among others. CRP is an acute phase protein, well established as a biomarker for infection and inflammation. High levels of CRP (40 to $200 \mu \mathrm{g} \mathrm{mL}^{-1}$ ) and PCT (above $0.5 \mathrm{ng} \mathrm{mL}^{-1}$ ) are known to be related with sepsis infection [5, 6]. However, both biomarkers are also affected by other non-inflammatory processes, such as burns or traumas. Interleukin-6 (IL6), together with TNF- $\alpha$ and IL-1 $\beta$, mediates the initial response of the innate immunity to injury or infection, enhancing the liver production of acute phase reactants, including CRP $[1,5]$. Additionally, circulating microRNAs (miRNAs) are acquiring importance as biomarkers for the non-invasive detection of diseases [7]. The evaluation of miRNAs differential expression levels in body fluids 
such as blood, saliva or urine can be a useful tool for diagnosis and prognosis. Some of them have been identified for playing a relevant role in the progression, diagnosis and staging of sepsis (i.e. miRNA15a, miRNA-146a, miRNA-16 or miRNA-223, among others) $[8-10]$.

Protein biomarkers like CRP and IL6 are individually analyzed following conventional techniques such as immunoassays or flow cytometry, which are precise and reliable, but they are also time consuming and require specialized personnel [11]. For the detection of circulating miRNAs, the most common techniques are real-time reversetranscription polymerase chain reaction (qRT-PCR), northern blot and fluorescent microarray technology. Due to the small size of the miRNAs and their low concentration in biological fluids ( $\mathrm{pM}-\mathrm{fM})$ these techniques need a large amount of sample, and sometimes lack sensitivity or robustness and are time-consuming [8].

Combining the detection of several of the most relevant biomarkers would increase the overall sensitivity and specificity of sepsis detection. Adding also the identification and quantification of the etiological infectious agent would ensure a more accurate diagnosis, a timely start of the appropriate antibiotic treatment and the improvement of the outcome of the sepsis process. Therefore, having a technology capable of detecting all these targets in a multiplexed analysis in a prompt, specific and simultaneous way, ideally at the patient's bedside or the ICU, might enable proper stratification of SIRS and sepsis patients.

Optical biosensors are ideal candidates for this purpose. Due to their integration capabilities they are excellent analytical tools to move the analysis from centralized laboratories to the point-of-care. Their design often offers fast turnaround times, easyto-handle features and in some cases, simultaneous detection capabilities [12]. Several optical devices using microarray formats to perform multiplexed assays have been previously proposed. Some of them are based on conventional labelled strategies (i.e. fluorescence or chemiluminescence) but others follow label-free approaches as the 
reflectometric interference spectroscopy, surface plasmon resonance imaging or the arrayed imaging reflectometry (AIR) [13-15]. Kemmler et al. [15] proposed a POC device for sepsis diagnosis based on a microarray using Total Internal Reflection Fluorescence (TIRF). The POC was employed to evaluate several sepsis biomarkers but required fluorescent labels and involved several fluid handling steps such as dilution, mixing, separation, pre-incubation and incubation to carry on sandwich and inhibition assays. Internal calibration in the biochip was also needed to prevent inaccuracy. Also, Mace et al. [16] used their developed AIR technology to detect various cytokine proteins involved in the body inflammatory response. Specific antibody-based macroarrays were manually generated. The design of the device allowed visualization and tracking of up to 8 differentiated spots, which limited its multiplexing potential and produced a high variability among measurements.

Recently, we presented a novel optical phase-sensitive interferometric biosensor based on microarray configuration as a new platform for high-throughput evaluation with potential of detecting specific biomarkers in blood plasma $[17,18]$. This POC has been developed within the framework of a Horizon 2020 European project (RAIS, www.raisproject.eu). The device has a small compact size $(20 \times 14 \times 23 \mathrm{~cm})$, is portable and user-friendly, giving results in around 1 minute. It requires $1 \mathrm{~cm}^{2}$ sensor chips and offers a large field of view $\left(20 \mathrm{~mm}^{2}\right)$. This feature facilitates the generation of dense arrays, which could allocate thousands of spots to be visualized simultaneously. We here demonstrate for the first time the feasibility of this POC device for the detection of protein and miRNA sepsis biomarkers. We have selected CRP and IL6 as protein targets, and miRNA-16 as miRNA model, being one of the several miRNAs with suspected diagnostic value to sepsis. We have developed and optimized all the biofunctionalization protocols and individual detection assays for each of those biomarkers. Moreover, we also show the multiplexed potential for the simultaneous 
detection of CRP and IL6, demonstrating the capabilities of our POC device for fast and label-free diagnosis of several biomarkers.

\section{Materials and Methods}

\section{Chemical and biological reagents}

Monoclonal antibody against CRP named ab183 (anti-CRP) was produced at and provided by DIESSE Diagnostica (Siena, Italy). Monoclonal antibody against IL6 (antiIL6) was purchased to Invitrogen (USA). CRP was obtained from BBI solutions (Cremlin, UK) and IL6 from Fitzgerald (Acton, USA).

All the buffer compounds, PBS $50 \mathrm{mM}(50 \mathrm{mM}$ phosphate buffer, $0.75 \mathrm{M} \mathrm{NaCl}, 2 \mathrm{mM}$ ethylenediaminetetraacetic acid (EDTA), pH 7), PBS $10 \mathrm{mM} \mathrm{(10} \mathrm{mM} \mathrm{phosphate} \mathrm{buffer,}$ $0.15 \mathrm{M} \mathrm{NaCl}, \mathrm{pH} 7), 5 x \mathrm{SSC}(0.75 \mathrm{M} \mathrm{NaCl}, 0.075 \mathrm{M}$ sodium citrate, $4 \mathrm{mM}$ EDTA, pH 7) were purchased from Sigma-Aldrich (Steinhem, Germany). Diethyl pyrocarbonate (DEPC), Protein G from Streptococcus sp. and bovine serum albumin (BSA) were purchased from Sigma-Aldrich (Steinhem, Germany). Oligo Analyzer software and RNA fold webserver was employed for secondary structure and self-annealing prediction of the probes and targets. DNA capture probes incorporating thiol (SH-DNA) at the 5'-end were purchased from IBIAN Technologies (Zaragoza, Spain) (see Table 1). Antibody against DNA/RNA duplex (Anti-DNA/RNA) was purchased from Kerafast (Boston, USA). All the buffers and other solutions for miRNA detection were prepared using DEPC- $\mathrm{H}_{2} \mathrm{O}$ (MilliQ water incubated overnight with $0.1 \%$ DEPC and autoclaved for $1 \mathrm{~h}$ at $121^{\circ} \mathrm{C}$ ). All solid plastic and glass materials were autoclaved for $1 \mathrm{~h}$ at $121^{\circ} \mathrm{C}$.

Table 1. DNA capture probes and miRNAs nucleotide sequences for miRNA assay.

\begin{tabular}{|l|l|l|}
\hline \multicolumn{1}{|c|}{ miRNA } & \multicolumn{1}{|c|}{ Sequence } & \multicolumn{1}{c|}{ DNA-SH capture probe } \\
\hline miRNA-16 & 5' UAGCAGCACGUAAAUAUUGGCG 3' & 5' SH-T 15 -CGCCAATATTTACGT 3' \\
\hline miRNA-21 & 5' UAGCUUAUCAGACUGAUGUUGA 3' & 5' SH-T 15-TCAACATCAGTCTGA 3' $^{\prime}$ \\
\hline miRNA-15a & & 5' SH-T 15 -CACAAACCATTATGT 3' \\
\hline
\end{tabular}

\section{Optical instrument, readout and data processing}


The POC device (dimensions: $20 \times 14 \times 23 \mathrm{~cm}$ ) has three main parts: a light source consisting of an external fiber-coupled LED $660 \mathrm{~nm}$ (Thorlabs), optical components (detailed in [17]), and an electronic assembly that controls the components and communicates with the computer. The LED light source was chosen to spectrally overlap with the transmission peak of the gold nanohole array (Au-NHA) used as plasmonic sensor chip [18]. The binding events occurring on the surface of Au-NHA chips were monitored with a custom designed LabView software which records the phase changes and renders an OPD (optical path difference) map image.

\section{Spotting of Au-NHAs chips}

All the spotting experiments carried out for the generation of the microarrays have been performed by the ICTS "NANBIOSIS", more specifically by the Unit of Biodeposition and Biodetection (U4) of CIBER in Bioengineering, Biomaterials \& Nanomedicine (CIBER-BBN) at ICN2 facilities. A dip-pen nanolithography-based spotter (BioForce Nanosciences, Utah, USA) was used, which allows the deposition of minute amounts of solutions in a very precise and custom-designed manner. Several microarrays generated over the sensor chip with different sizes such as $8 \times 8$ or $10 \times 10$ spots (with an average diameter of around $55 \mu \mathrm{m}$ and an array pitch of $250 \mu \mathrm{m}$ ) were routinely generated throughout all the experiments.

\section{Plasmonic Gold Nanohole Array chips}

Fabrication of the plasmonic sensor chips was done in a clean room using wafer-scale and high-throughput fabrication methods. Fused silica wafers were coated with $\mathrm{Ti} / \mathrm{Au}$ $(10 / 120 \mathrm{~nm})$ and patterned with $200 \mathrm{~nm}$ diameter and $600 \mathrm{~nm}$ period nanohole arrays. The fabrication process is explained in detail in [18]. Before use, the Au-NHA chips $\left(1 \times 1 \mathrm{~cm}^{2}\right)$ were cleaned by consecutive heating at $50^{\circ}$ and sonicating $(1 \mathrm{~min})$ with solvents of increasing polarity (i.e. acetone, ethanol and MilliQ water). Then, they were dried with a $\mathrm{N}_{2}$ flow and placed in a $\mathrm{UV} / \mathrm{O}_{3}$ chamber (Bioforce Nanoscience, Utah, 
USA, included in the Unit of Biodeposition and Biodetection (U4), from the ICTS "NANBIOSIS") for $30 \mathrm{~min}$. The sensor chips were finally rinsed with ethanol and MilliQ water and dried with $\mathrm{N}_{2}$ flow.

\section{Biofunctionalization and protein measurements with Au- NHA chips}

The attachment of protein bioreceptors (antibodies) to the Au-NHA chips was done via Protein G capture to facilitate the orientation of the antibodies. Briefly, Protein G (500 $\mu \mathrm{g} \mathrm{mL} \mathrm{m}^{-1}$ in PBS $10 \mathrm{mM}$ with $5 \%$ glycerol) was attached through non-covalent physisorption by direct spotting over clean Au-NHA sensor chips. The sensor chips were incubated for $2 \mathrm{~h}$ at room temperature $(\mathrm{RT})$ and rinsed with PBS to remove unbound protein G. The sensor chips were incubated with a BSA solution (1\% in PBS $10 \mathrm{mM}, 1 \mathrm{~h}$ at $\mathrm{RT}$ ) to block remaining bare gold area and were rinsed again with PBS. The chips were then incubated with the antibody $\left(250 \mu \mathrm{g} \mathrm{mL}^{-1}\right.$ in PBS $10 \mathrm{mM}, 2 \mathrm{~h}$ at RT) and finally rinsed with PBS and dried with $\mathrm{N}_{2}$. Calibration curves were obtained by evaluating different known concentrations of CRP or IL6 in triplicates (from 10 to 500 $\mu \mathrm{g} \mathrm{mL} \mathrm{m}^{-1}$ dissolved in PBS). Each step of the biofunctionalization was monitored by rinsing and drying the sensor chips and placing them in the POC device to obtain the OPD. The Limit of Detection (LoD) was calculated as three times the standard deviation of the signal corresponding to a blank sample.

The multiplexed test was done by immobilization of both anti-CRP and anti-IL6 antibodies. A sensor chip was divided in four sections. An $8 \times 8$ microarrays of Protein $G$ was generated in each one of them. The four differentiated areas were separated by means of a hydrophobic pen that created a hydrophobic barrier that avoided sample mixing. Blocking with BSA (1\%) for 30 min was also performed. Then two of the microarrays were incubated with anti-IL6 and two with anti-CRP antibodies (see Figure 5). The sensor chip was then incubated with solutions containing either CRP, IL6 or a mixture of both $\left(200 \mu \mathrm{g} \mathrm{mL}^{-1}\right)$. 


\section{Biofunctionalization and miRNA measurements with Au- NHA chips}

Biofunctionalization for miRNA detection was achieved via thiol-gold linkage by spotting a solution of SH-DNA capture probes (50 $\mu \mathrm{M}$ in PBS $50 \mathrm{mM}$ with $10 \%$ glycerol) over clean Au-NHAs chips and incubating them overnight at RT. The sensor chips were rinsed with DEPC- $\mathrm{H}_{2} \mathrm{O}$ and incubated with $\mathrm{BSA}(0.1 \%$ in PBS $10 \mathrm{mM}$ for $30 \mathrm{~min})$ to block remaining gold area. The sensor chips were rinsed with $\mathrm{DEPC}-\mathrm{H}_{2} \mathrm{O}$ water and dried with $\mathrm{N}_{2}$. Target miRNA (miRNA-16) at different concentrations $(0.1-50 \mu \mathrm{M}$ in $5 \mathrm{x}$ SSC buffer) was incubated with DNA spotted sensor chips for $1 \mathrm{~h}$ at RT. After rinsing, the sensor chips were incubated with anti-DNA/RNA (at a fixed concentration of $80 \mu \mathrm{g}$ $\mathrm{mL}^{-1}$ diluted in $0.5 \mathrm{xSS}$ ) during $45 \mathrm{~min}$ at RT. Finally, the sensor chips were rinsed with DEPC- $\mathrm{H}_{2} \mathrm{O}$ water and dried with $\mathrm{N}_{2}$ flux. Each step of the biofunctionalization and detection was monitored by placing the rinsed and dried sensor chips in the POC device to obtain the OPD. Calibration curves were obtained by measuring different standards of known concentration of target miRNA-16 by triplicate.

\section{Calculation of the optical path difference (OPD)}

Each step of the assay was monitored by tracking the OPD signals of the spots. Upon binding of analyte, the total OPD of the spot increases. The plotted signal is the result of the difference in OPD value before $\left(O P D_{t_{0}}\right)$ and after incubation $\left(O P D_{t_{t}}\right)$ with the target analyte $\left(\triangle O P D=O P D_{t_{t}}-O P D_{t_{0}}\right)$. Calibration curves were fitted to a non-linear one site total binding equation (CRP and miRNA-16) or to a linear fitting (IL6). The $\triangle \mathrm{OPD}$ and the standard deviation (SD) of each concentration were plotted versus the target concentration.

\section{Results and Discussion}

POC biosensor 
The POC biosensor device has been previously described [17]. It is based on a lensfree interferometric microscopy which evaluates changes in the topography of transparent surfaces, allowing direct observation of target-binding events. Briefly, the device is an optical microarray reader with large-field-of-view $\left(20 \mathrm{~mm}^{2}\right)$ (FOV), in which a polarized light beam is sheared into two beams (reference and signal, see Figure 1a) that pass through the sample. These two beams are then recombined and the final output light is recorded by a CMOS sensor. Changes in the phase of the light beams when passing through a transparent substrate create an interferometric pattern over the full FOV of the camera which is translated into an OPD map. Thus, local deviations in refractive index and thickness (such as biomarkers present in a sample) can be tracked by a camera and then processed by an algorithm to extract the OPD. The algorithm isolates each spot and its surroundings and calculates the difference between the maximum and the minimum phase-shift values found in that area, rendering the final OPD value for each spot. These light phase-shifts can be enhanced by the employment of sensor surfaces such as those based on metallic nanostructures, compatible with the working principle of the device. The present of the nanoplasmonic structures boosts the sensitivity and improves the feasibility of the POC device for biosensing applications, as recently demonstrated with gold nanohole arrays (Au-NHA) [18]. The generation of microarray-based spotted surface and custom-designed biofunctionalization over the surface of the Au-NHA sensor chips can be done with conventional molecular printers such as a dip-pen nanolithography instrument (Figure 1b) which ensures the appropriate spot size and high spatial accuracy in well-defined patterns. The variation of OPD ( $\triangle \mathrm{OPD})$ upon binding can therefore be correlated with the analyte concentration (Figure 1c). In this format, each spot can eventually be related to a specific biomarker, allowing the immobilization of multiple bioreceptors to target different sepsis biomarkers.

\section{CRP and IL6 immunodetection assay}


To evaluate CRP and IL6 with the POC biosensor, we designed a direct and label-free immunoassay strategy, using antibodies as specific bioreceptors immobilized on the sensor chip surface. Direct assays are always the desired option, as they involve a simple one-step format, which reduces time and hands-on involvement, and facilitates further automation for POC deployment. In order to maximize the efficiency of the assay, the antibody should be as exposed as possible to facilitate the target recognition. Hence, a biofunctionalization based on the use of Protein $\mathrm{G}$ as antibodyorienting molecule was attempted. The crystallizable fraction $(\mathrm{Fc})$ of the antibodies interacts with Protein G forming a strong but reversible binding, thus leaving the Fab part, which contains the recognition binding sites, facing outwards. The array distribution was achieved by directly spotting Protein $\mathrm{G}$ solution $\left(500 \mu \mathrm{g} \mathrm{mL}^{-1}\right)$ onto clean bare sensor chips (Figure 2a). The protein anchors to the gold surface via physisorption. Lower concentrations of Protein G were tested leading to less amount of antibody adsorbed (i.e. low $\triangle$ OPD which introduced higher variability among spots). In order to minimize further adsorptions onto the remaining gold areas in subsequent steps, a blocking step was introduced. Skim milk, antifouling PEGylated compounds (i.e. PLL-PEG) and BSA were tested as blocking agents. However, only when using $1 \%$ BSA blocked surfaces, a significant and reproducible attachment of the specific antibody (anti-CRP or anti-IL6 at $250 \mu \mathrm{g} \mathrm{mL}^{-1}$ ) onto the Protein $\mathrm{G}$ spots was observed (i.e. higher $\triangle \mathrm{OPD}$, see Figure $\mathbf{2 c}$ as a representative example). The other blocking agents probably interacted with the Protein G, preventing the subsequent binding of the antibody. For this reason, blocking with BSA was selected for further experiments. The mean of 64 spots was measured for $O P D_{t_{0}}$ and for $O P D_{t_{t}}$ as working with several averaged OPD values reduced variability and improved reliability of the measurements. Figure $\mathbf{2 b}$ shows the mean OPD values obtained in several sensor chips $(n=30)$ for anti-CRP and anti-IL6 arrays, respectively. The same OPD pattern was observed in both cases with very similar overall signals and excellent reproducibility according to the SD obtained. Protein G was physically adsorbed on the Au-NHA chip, resulting in 
mean OPD values around 22. After the BSA blocking, the OPD is reduced between 3-4 units, which suggests that a layer of BSA was created over the gold surface attaching to the free remaining gold areas of the sensor chip. The addition of a monolayer of BSA changes the surface reference (i.e. it increases the OPD as a result of the adsorption), thus, reducing the $\triangle O P D$ between the protein $G$ spots and the surrounding area. As can be seen, the intensity increases considerably after incubation with the antibody (OPD values of $35.7 \pm 5.4$ and $40.00 \pm 5.1$ for anti-CRP and anti-IL6 respectively), which confirms the preferential avidity of antibody for the immobilized Protein G. It is worth noting that other ways of anchoring antibodies were tested, such as covering the whole surface with a Protein G layer (through its covalent attachment to a selfassembled monolayer) followed by antibody spotting or by also spotting biotinylated antibodies over a streptavidin coated layer. However, the antibody coated sensor chips showed less reproducibility and overall lower OPD signals (i.e. less number of receptors on the surface) which resulted in biofunctionalized sensor chips with worst performance (data not shown).

The specificity provided by the chosen strategy was assessed with different controls as summarized in the Figure 3 for the CRP assay. We observed that the addition of the target protein resulted in a clear signal enhancement $((\triangle O P D=8.83$, in Figure $\mathbf{3}(\mathbf{1}))$. However, no OPD increase was observed if the array was incubated with a different protein (in Figure $3(2)$ ). Similarly, incubation of CRP over a non-specific antibody resulted in negligible increase in the OPD $(\triangle O P D=-1.47)$ (see Figure 3(3)). Moreover, in the absence of any antibody (i.e. only spotted Protein G arrays and BSA) the CRP did not bind (Figure 3(4) corroborating the lack of non-specific binding over the blocked surface. These different tests confirmed that the signal corresponds exclusively to the specific recognition of the protein for its specific antibody (in this case, CRP and antiCRP, but extendable to any other antigen-antibody pair). 
With the aforementioned conditions the corresponding calibration curves for both CRP and IL6 were obtained by incubating several arrays (8x8 spots) with different target concentrations (Figure 4a and c). The calibration curve for CRP showed a linear concentration-dependent region before reaching a saturation. A limit of detection (LoD) of $18 \mu \mathrm{g} \mathrm{mL} \mathrm{m}^{-1}$ was estimated. Since CRP levels in blood plasma of healthy individuals are commonly found below $10 \mu \mathrm{g} \mathrm{mL} \mathrm{m}^{-1}$ and can drastically increase to around $300 \mu \mathrm{g}$ $\mathrm{mL}^{-1}$ in patients with severe infection [19], our approach can comfortably allow the detection of this protein in any infection particularly, sepsis. In the case of IL6, a linear response was observed without reaching saturation for the range of concentrations analyzed. Although it showed a good fitting $\left(R^{2}=0.9647\right)$ the LoD was relatively high $\left(\mathrm{LoD}=88 \mu \mathrm{g} \mathrm{mL} \mathrm{L}^{-1}\right)$ for the requirements for sepsis diagnosis. The affinity of the antibody might have an influence in this lower level of detectability compared for instance with CRP. However, this can be also associated to the much lower MW of IL6, since the working principle of the device is related to the refractive index changes on the surface, which in turn is related to the mass (as described in the experimental section and ref [17]).

In order to establish the accuracy of the method, some spiked blank samples for both biomarkers that fit within the linear range of the curve were prepared and analyzed with the biosensor. Figure $\mathbf{4 b}$ and $d$ shows the correlation of concentrations with spiked samples. The slopes of the corresponding linear fittings are 0.817 for CRP and 0.996 for IL6, respectively. According to this, IL6 assay, with a slope almost equal to 1, shows an excellent accuracy. In the case of CRP there is a slight underestimation probably due to the high concentration range close to the saturation area (i.e. above $200 \mu \mathrm{g} \mathrm{m} \mathrm{L}^{-}$ ${ }^{1}$ ). Narrowing down the quantification to the lower concentration range (up to $100 \mu \mathrm{g}$ $\mathrm{mL}^{-1}$ ) can ensure a higher level of accuracy.

\section{CRP and IL6 multiplexed detection}


Based on these results, the simultaneous detection of both CRP and IL6 in the same sensor chip was attempted to demonstrate the feasibility of performing multiplexed analysis with the POC device. Gold NHA sensor chips were prepared as depicted in the Figure 5a. Four $8 \times 8$ microarrays were spatially distributed to facilitate further incubation with both the antibodies in the biofunctionalization step and the biomarkers for the detection. Two microarrays were functionalized with anti-CRP and the other two with anti-IL6. Finally, both targets were incubated either individually or together in one solution. Two different target concentrations were evaluated (100 and $200 \mu \mathrm{g} \mathrm{mL}^{-1}$ ). The results are summarized in Figure 5a. When tested individually, only in the microarrays where the protein was incubated with its specific antibody, an increase in the OPD was observed, which confirms the specificity of both assays. When analyzed together as a single solution containing both CRP and IL-6 at the same concentration $\left(200 \mu \mathrm{g} \mathrm{mL}^{-1}\right)$ we observed similar $\triangle$ OPDs (see Figure $5 \mathbf{b}$ ), although slightly higher for CRP compared with the same concentration in a solution only containing this protein. A possible reason for this behavior could be related to the high concentration of CRP used in this experiment (i.e. $200 \mu \mathrm{g} \mathrm{mL} \mathrm{m}^{-1}$ of $\mathrm{CRP}$ in $10 \mu \mathrm{L}$ ) which, according to our experience, resulted in the formation of the so-called "coffee ring effect" on the spots, affecting the data processing to analyze the OPD. These proof-of-concept experiments though with a limited degree of multiplexing, exemplifies the potential of the biofunctionalization strategy and the POC biosensor for the simultaneous analysis of at least four different proteins in a single chip (i.e. in a 4-array design like the one shown in Figure 5).

\section{miRNA detection assay}

In the case of miRNA assays, a complementary DNA probe to the target sequence was designed to specifically detect miRNA-16 via DNA/RNA hybridization. A thiol group was

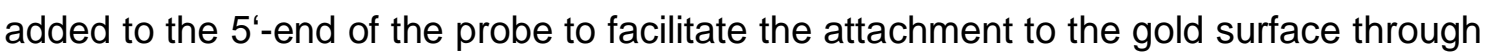
the formation of stable self-assembled monolayers (SAM) [20]. A poly thymine 
sequence with 15 thymines $\left(T_{15}\right)$ was introduced after the thiol group to move away the complementary part from the gold surface (see Table 1). The biofunctionalization process (Figure 6a) involved the spotting of the thiolated DNA probes to generate the array followed by a blocking step of the remaining gold area with BSA. In this case, given the relatively small size of the miRNA $(\mathrm{MW}=6.6 \mathrm{kDa})$ an amplification step was considered by using an antibody that specifically binds only to DNA/RNA duplexes [21]. This anti-DNA/RNA antibody does not cross-react with double stranded DNA, double stranded RNA, or single DNA and RNA. The considerably high molecular weight of the antibodies $(\mathrm{MW}=115 \mathrm{kDa})$ triggers a larger phase change, which in turn enhances the overall signal and improves the overall sensitivity (Figure 6b, c).

The immobilization steps showed similar behavior regardless of the DNA probe immobilized as can be seen in Figure $\mathbf{6 d}$ where similar signals were obtained for three different sequences (i.e. between $20-25$ bases). The great affinity between the gold and thiol permits a simple, fast, direct one-step immobilization. As in the protein-based assay, the BSA blocking step also resulted in an OPD decrease consistent with its adsorption over gold (see Figure $\mathbf{6 d}$ ). As can be seen in Figure $\mathbf{6 c}$, the direct detection of miRNA was hardly observed, while after the addition of the specific anti-DNA/RNA the OPD enhancement was easily detectable. The specificity provided by the capture probe and the selectivity of the immobilization and blocking strategy were assessed with two negative control experiments. Either a non-complementary miRNA (miRNA21) or simply buffer without miRNA were incubated in the hybridization step, followed by the addition of the anti-DNA/RNA. As shown in the Figure 7a (1) a significantly higher enhancement was observed for the specific target $(\triangle \mathrm{OPD}=7.06$ for the antibody amplification step). However, no significant OPD increase resulted when a non-complementary miRNA was present Figure $7 a(2)$ or in the absence of any miRNA

Figure 7a (3), concluding that no DNA/RNA hybrids are formed, and corroborating the absence of nonspecific binding of anti-DNA/RNA over the functionalized array. 
With the conditions previously detailed, a calibration curve for miRNA-16 was obtained (Figure 7b). The curve shows the signal obtained in the amplification step with the antiDNA/RNA. Also, in this case, a saturation region is observed for some of the miRNA concentrations analyzed. The LoD obtained was around $1 \mu \mathrm{M}$; this value is limited for the clinical requirements of this family of biomarkers, which commonly are within the $\mathrm{nM}$ - $\mathrm{fM}$ range in serum or plasma [8]. However, the developed assay using a sequence of DNA as bioreceptor offers a fast, straightforward label-free methodology for miRNA detection and illustrates the versatility of the POC device to measure not only proteins but also oligonucleotides.

In general terms, the sensitivity level could be further enhanced by additional amplification strategies which increased the overall mass attached to the surface. Thus, for the protein biomarkers, the addition of a second antibody in a sandwichbased assay, either free or even conjugated to large entities such as nanoparticles could be a feasible and commonly used option. This would be also possible for the miRNA detection by directly coupling the ant-DNA/RNA antibody to the same kind of nanoparticles, which would eventually increase even more the signal.

\section{Conclusions}

A recently developed $\mathrm{POC}$ device based on lens-free microscopy and light interferometry has been applied to the detection of specific biomarkers of different nature (proteins and miRNAs) with relevance in the diagnosis of sepsis. CRP and IL6 proteins and miRNA-16 have been selected as representative biomarkers whose detection in plasma can help diagnose sepsis in a fast and reproducible way. The three assays have been optimized and are based on direct sample detection for proteins and require an additional amplification step for the miRNAs. The sample analysis is fast (it takes around $1 \mathrm{~min}$ after sample incubation). The LoDs obtained for the proteins are in the range of $\mu \mathrm{g} \mathrm{mL}^{-1}$ and for miRNA in the low $\mu \mathrm{M}$, which may be enough for CRP in sepsis diagnosis but should be enhanced for smaller biomarkers such as IL6 and 
miRNAs. Improvements in the POC device such as the use of other nanoplasmonic structures which improve the capabilities of current configuration, or the incorporation of multispectral source to accomplish other sharper modes might be possible options to enhance the device performance. The strategies developed are reproducible and show excellent specificity, which make them highly suitable for further improvement and implementation in multiplexing configurations. The demonstration shown here with two protein biomarkers highlights the versatility of the technology. Current biofuntionalization strategy allows four different antibodies to be immobilized in a single chip (i.e. antibodies are not directly spotted but incubated over four different Protein G arrays distributed in the $1 \mathrm{~cm}^{2}$ chip surface). This could be expanded by making use of microfluidics which facilitated both the integration of more densely packed arrays and their subsequent antibody immobilization, enabling a larger level of throughput screening in a single measurement. Furthermore, the compactness and autonomy of the device paves the way towards its penetration in different decentralized environments, such as the hospital benchtop, at the physician desk or even at ICU unit for sepsis diagnosis or for other diseases.

\section{Acknowledgments}

We thank the financial support from European Union's Horizon 2020 Research and Innovation program (RAIS Project, grant agreement 644956). We thank Diesse diagnostica senese SPA for providing anti-CRP antibody antibodies. The ICN2 is funded by the CERCA programme / Generalitat de Catalunya. ICN2 is supported by the Severo Ochoa program from Spanish MINECO (Grant No. SEV-2017-0706). ICN2 thanks FEDER funds from the European Regional Development Funds program (Project FICTS-1420-27 selected by MINECO). LD acknowledges financial support funded by the SNSF (Swiss National Science Foundation) through an Early Postdoc.Mobility Fellowship (No. 178438). RT, LD and VP acknowledge financial support from the Spanish Ministry of Economy and Competitiveness through the 
Severo Ochoa Programme for Centres of Excellence in R\&D (SEV-2015-0522) and OPTO-SCREEN (TEC2016-75080-R), from Fundacio Privada Cellex, from Generalitat de Catalunya through the CERCA program and AGAUR 2017 SGR 1634.

\section{References}

[1] K. Essandoh, G.-C. Fan, Role of extracellular and intracellular microRNAs in sepsis, Biochim. Biophys. Acta - Mol. Basis Dis. 1842 (2014) 2155-2162.

[2] D.C. Angus, W.T. Linde-Zwirble, J. Lidicker, G. Clermont, J. Carcillo, M.R. Pinsky, Epidemiology of severe sepsis in the United States: analysis of incidence, outcome, and associated costs of care., Crit. Care Med. 29 (2001) 1303-1310.

[3] M.M. Levy, A. Artigas, G.S. Phillips, A. Rhodes, R. Beale, T. Osborn, J.L. Vincent, S. Townsend, S. Lemeshow, R.P. Dellinger, Outcomes of the Surviving Sepsis Campaign in intensive care units in the USA and Europe: A prospective cohort study, Lancet Infect. Dis. 12 (2012) 919-924.

[4] M. Prucha, G. Bellingan, R. Zazula, Sepsis biomarkers, Clin. Chim. Acta 440 (2015) 97-103.

[5] J.D. Faix, Biomarkers of Sepsis, Crit. Rev. Clin. Lab. Sci. 50 (2013) 23-26.

[6] B. Clyne, J.S. Olshaker, The C-reactive protein, J. Emerg. Med. 17 (1999) 1019-1025.

[7] J. Huang, Z. Sun, W. Yan, Y. Zhu, Y. Lin, J. Chen, B. Shen, J. Wang, Identification of MicroRNA as sepsis biomarker based on miRNAs regulatory network analysis, Biomed Res. Int. 2014 (2014) 594350.

[8] A. Hruštincová, H. Votavová, M.D. Merkerová, Circulating microRNAs: Methodological aspects in detection of these biomarkers, Folia Biol. 61 (2015) 2013218.

[9] H. Wang, P. Zhang, W. Chen, D. Feng, Y. Jia, L. Xie, Four serum microRNAs identified as diagnostic biomarkers of sepsis, J. Trauma Acute Care Surg. 73 (2012) 850-854.

[10] S. Caserta, F. Kern, J. Cohen, S. Drage, S.F. Newbury, M.J. Llewelyn, Circulating Plasma microRNAs can differentiate Human Sepsis and Systemic Inflammatory Response Syndrome (SIRS), Sci. Rep. 6 (2016) 1-13.

[11] P. Salvo, V. Dini, A. Kirchhain, A. Janowska, T. Oranges, A. Chiricozzi, T. Lomonaco, F. Di Francesco, M. Romanelli, Sensors and biosensors for C-reactive protein, temperature and $\mathrm{pH}$, and their applications for monitoring wound healing: $\mathrm{A}$ review, Sensors 17 (2017) 2952.

[12] D. Xu, X. Huang, J. Guo, X. Ma, Automatic smartphone-based microfluidic biosensor system at the point of care, Biosens. Bioelectron. 110 (2018) 78-88. 
[13] R. Duer, R. Lund, R. Tanaka, D.A. Christensen, J.N. Herron, In-plane parallel scanning: A microarray technology for point-of-care testing, Anal. Chem., 82 (2010) 8856-8865.

[14] C.R. Mace, C.C. Striemer, B.L. Miller, Theoretical and experimental analysis of arrayed imaging reflectometry as a sensitive proteomics technique, Anal. Chem. 78 (2006) 5578-5583.

[15] M. Kemmler, U. Sauer, E. Schleicher, C. Preininger, A. Brandenburg, Biochip point-of-care device for sepsis diagnostics, Sens. Act. B 192 (2014) 205-215.

[16] C.R. Mace, C.C. Striemer, B.L. Miller, Detection of human proteins using arrayed imaging reflectometry, Biosens. Bioelectron. 24 (2008) 334-337.

[17] R.A. Terborg, J. Pello, I. Mannelli, J.P. Torres, V. Pruneri, Ultrasensitive interferometric on-chip microscopy of transparent objects, Sci. Adv., 2 (2016) e1600077.

[18] F. Yesilkoy, R. A.Terborg, J. Pello, A. Belushkin, Y. Jahani, H. Altug, V. Pruneri, Phase-sensitive plasmonic biosensor using a portable and large field of view interferometric microarray imager, Light Sci. Appl., 7 (2018) 17152.

[19] P. Buchegger, C. Preininger, Four assay designs and on-chip calibration: Gadgets for a sepsis protein array, Anal. Chem. 86 (2014) 3174-3180.

[20] Y. Sakao, F. Nakamura, N. Ueno, M. Hara, Hybridization of oligonucleotide by using DNA self-assembled monolayer, Colloid Surf.-B Biointerfaces 40 (2005) 149152.

[21] S.J. Boguslawski, D.E. Smith, M.A. Michalak, K.E. Mickelson, C.O. Yehle, W.L. Patterson, R.J. Carrico, Characterization of monoclonal antibody to DNA - RNA and its application to immunodetection of hybrids, J. Immunol. Methods, 89 (1986) 123-130. 
a

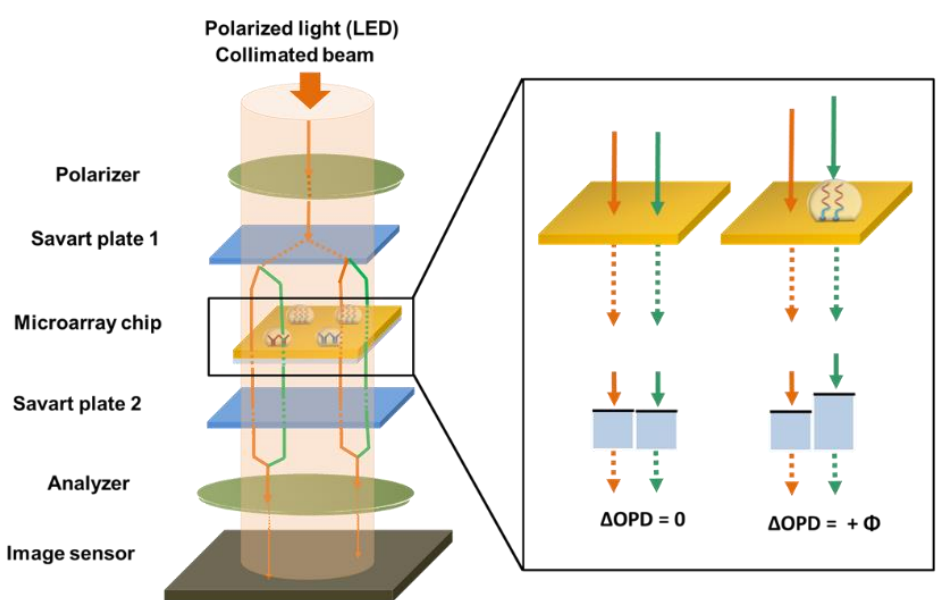

b

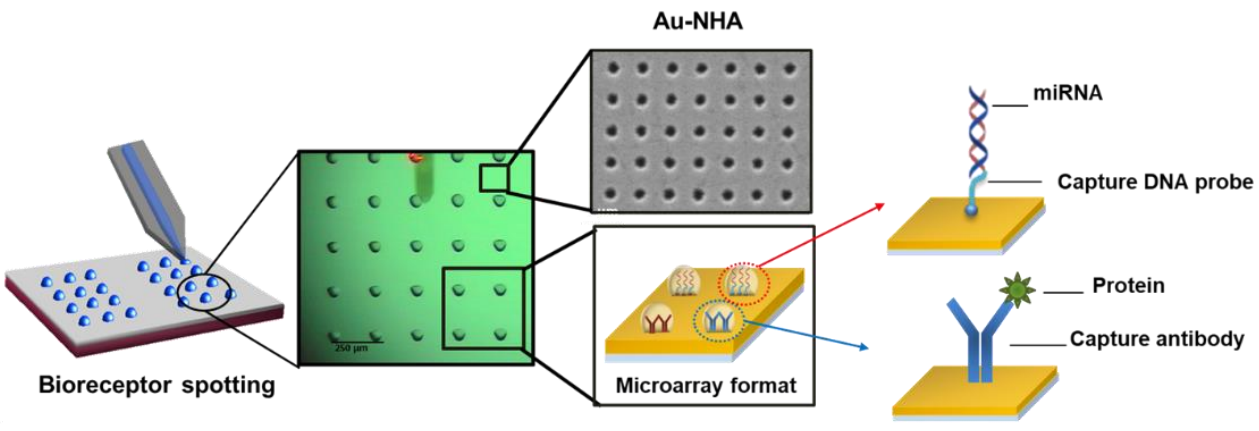

c
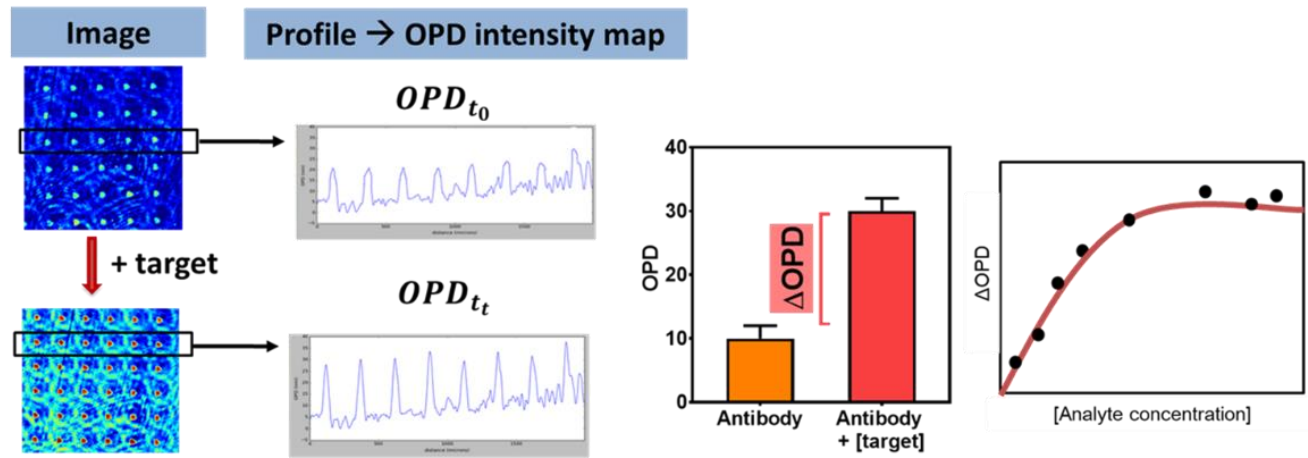

[Analyte concentration]

Figure 1: Scheme showing the sequential steps followed for the immobilization process and detection with the POC device. a) Optical principle of the device. $\Phi=$ Phase difference ([17], adapted). b) Biofunctionalization of the Au-NHA chips using a dip-pen nanolithography molecular printer. A microarray image with different drops with a diameter of $55 \mu \mathrm{m}$ and a SEM image of the nanoplasmonic chip are shown. c) Images of the arrays generated after software processing. Differences in the OPD $\left(\triangle O P D=O P D_{t_{t}}-O P D_{t_{0}}\right)$ in each spot with and without the target can be related with the analyte concentration. 
a

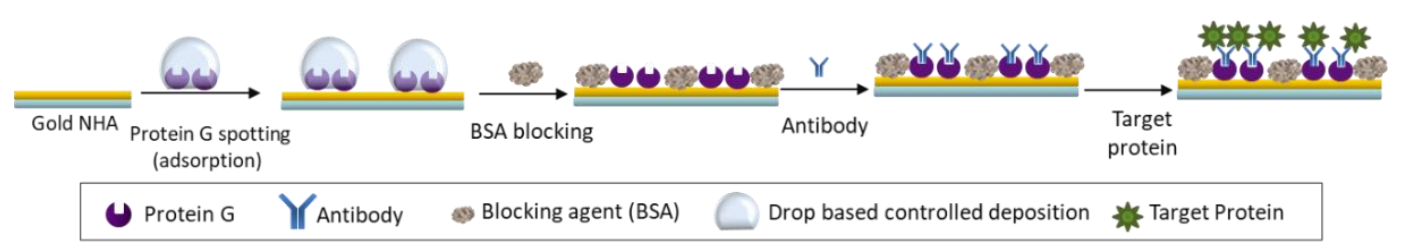

b

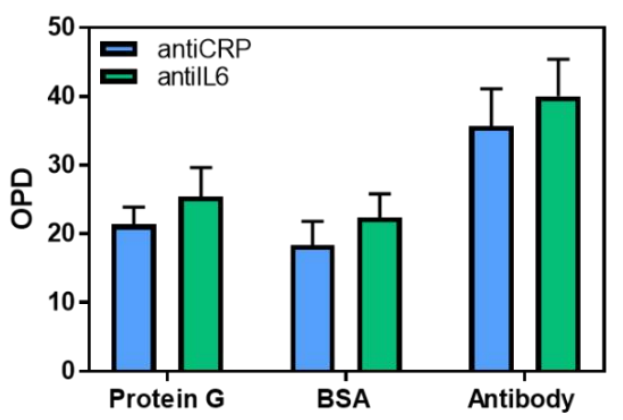

C

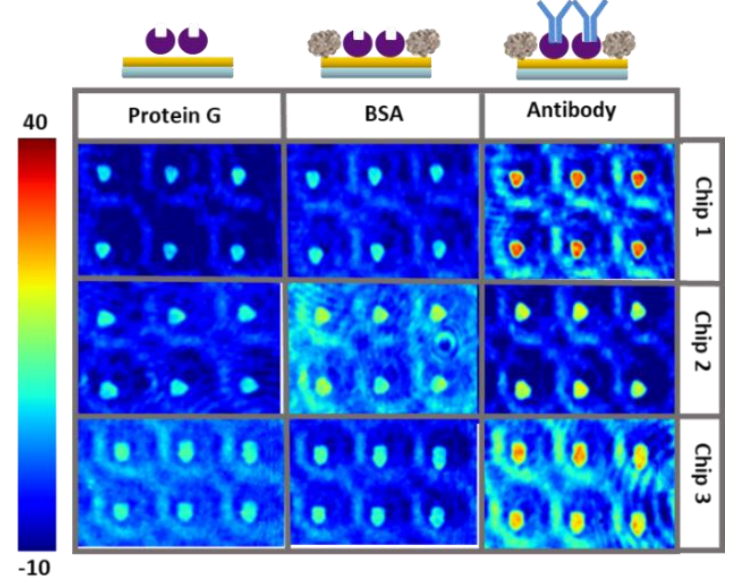

Figure 2: Protein detection immunoassay a) Scheme of the immobilization involving Protein $\mathrm{G}$ physisorption, antibody binding, blocking of gold areas and detection of CRP or IL6. b) Averaged OPD values obtained in each step for 30 chips for both target proteins. c) Representative intensity OPD maps of microarrays obtained in three different chips, showing the three steps of the antibodies biofunctionalization protocol. 


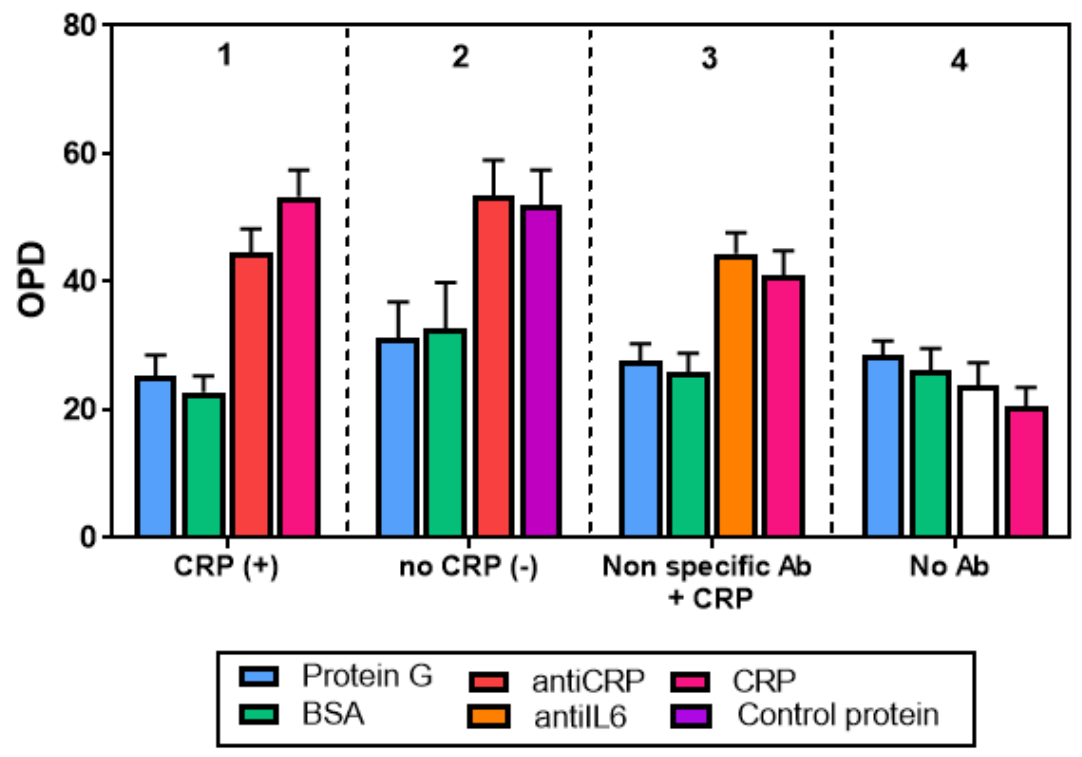

Figure 3: Specificity study for the CRP assay showing the variation in the OPD in 4 different arrays: (1) Positive control ([CRP] $=500 \mu \mathrm{g} \mathrm{mL}^{-1}$ ) incubated on an anti-CRP functionalized array; (2) Control protein $\left(200 \mu \mathrm{g} \mathrm{mL}^{-1}\right)$ incubated on an anti-CRP functionalized array; (3) CRP (500 $\mu \mathrm{g} \mathrm{mL}^{-1}$ ) incubated on array functionalized with a non-specific antibody; (4) CRP (500 $\mu \mathrm{g} \mathrm{mL}^{-1}$ ) incubated on unmodified array (i.e. only Protein G spots on a BSA blocked surface). 


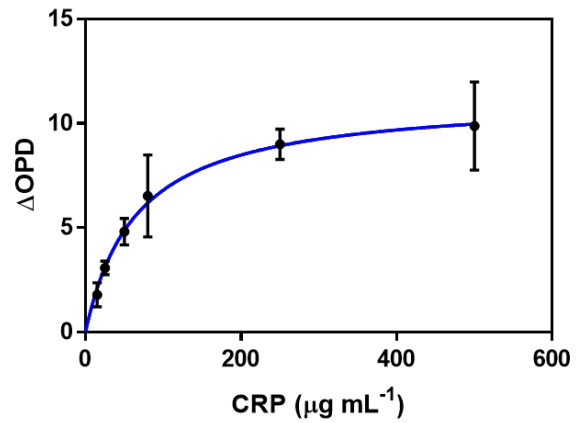

C

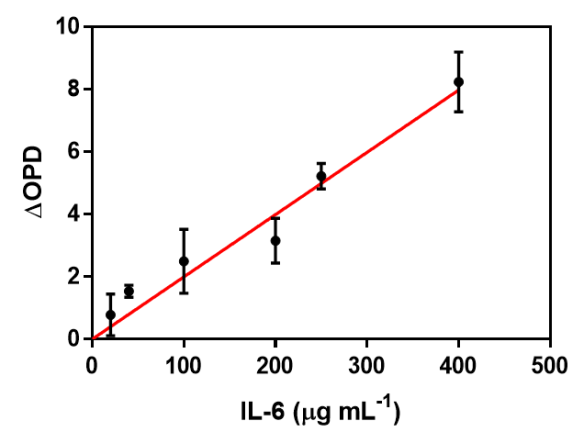

b

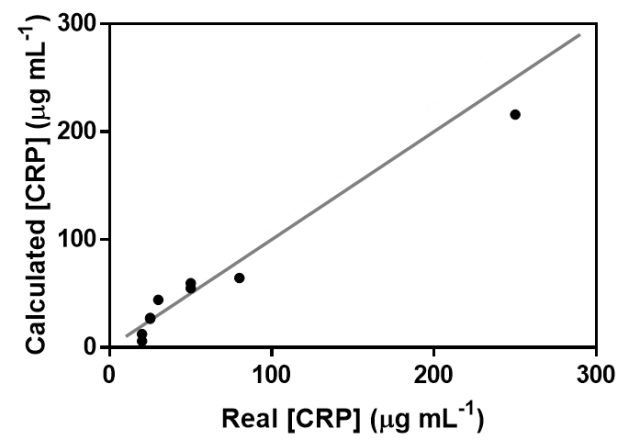

d

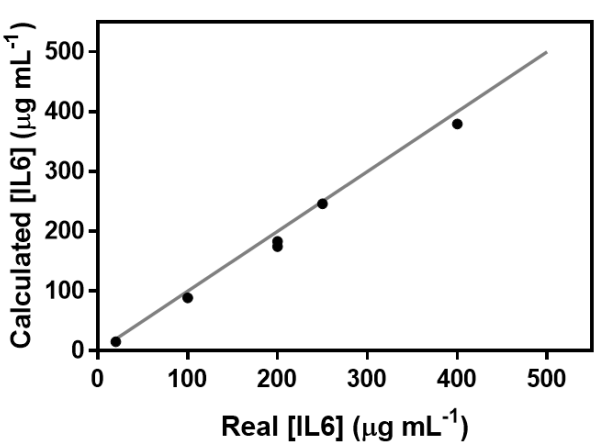

Figure 4: Calibration curves in PBS for (a) CRP and (c) IL6 obtained with the POC device. Correlation graphs for blind (b) CRP and (d) IL6 spiked PBS samples. All data show mean $\triangle \mathrm{OPD} \pm \mathrm{SD}$ of triplicate measurements. 


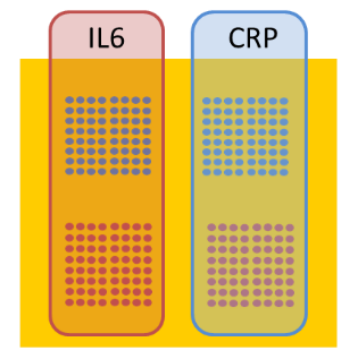

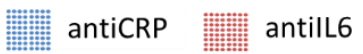

b

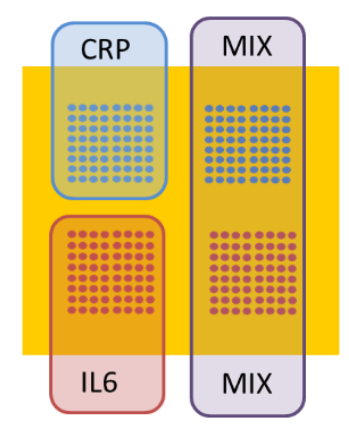

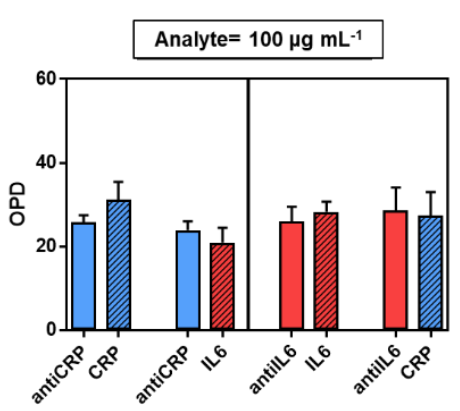
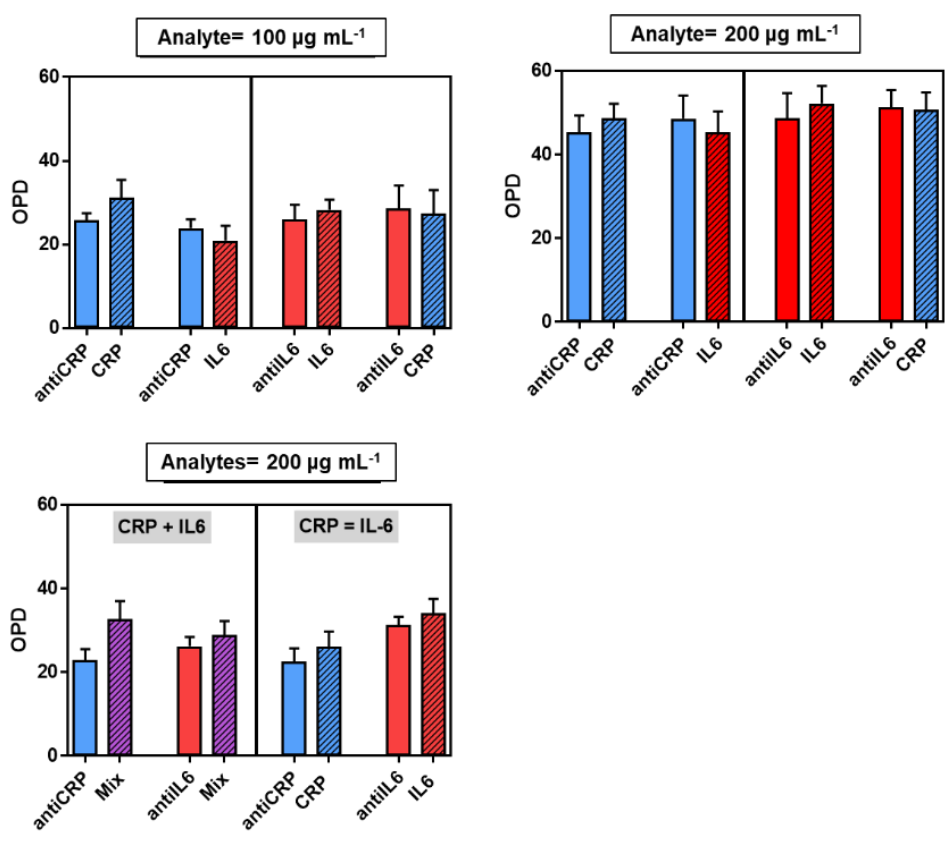

Figure 5: Multiplexed measurements of CRP and IL6 with the POC device. a) Incubation of arrays with solutions containing only one biomarker (CRP or IL6) at $100 \mu \mathrm{g} \mathrm{m}^{-1}$ and $200 \mu \mathrm{g}$ $\mathrm{mL}^{-1}$.

b) Incubation of arrays with a solution containing both CRP and IL6 biomarkers at a concentration of $200 \mu \mathrm{g} \mathrm{mL}^{-1}$. 

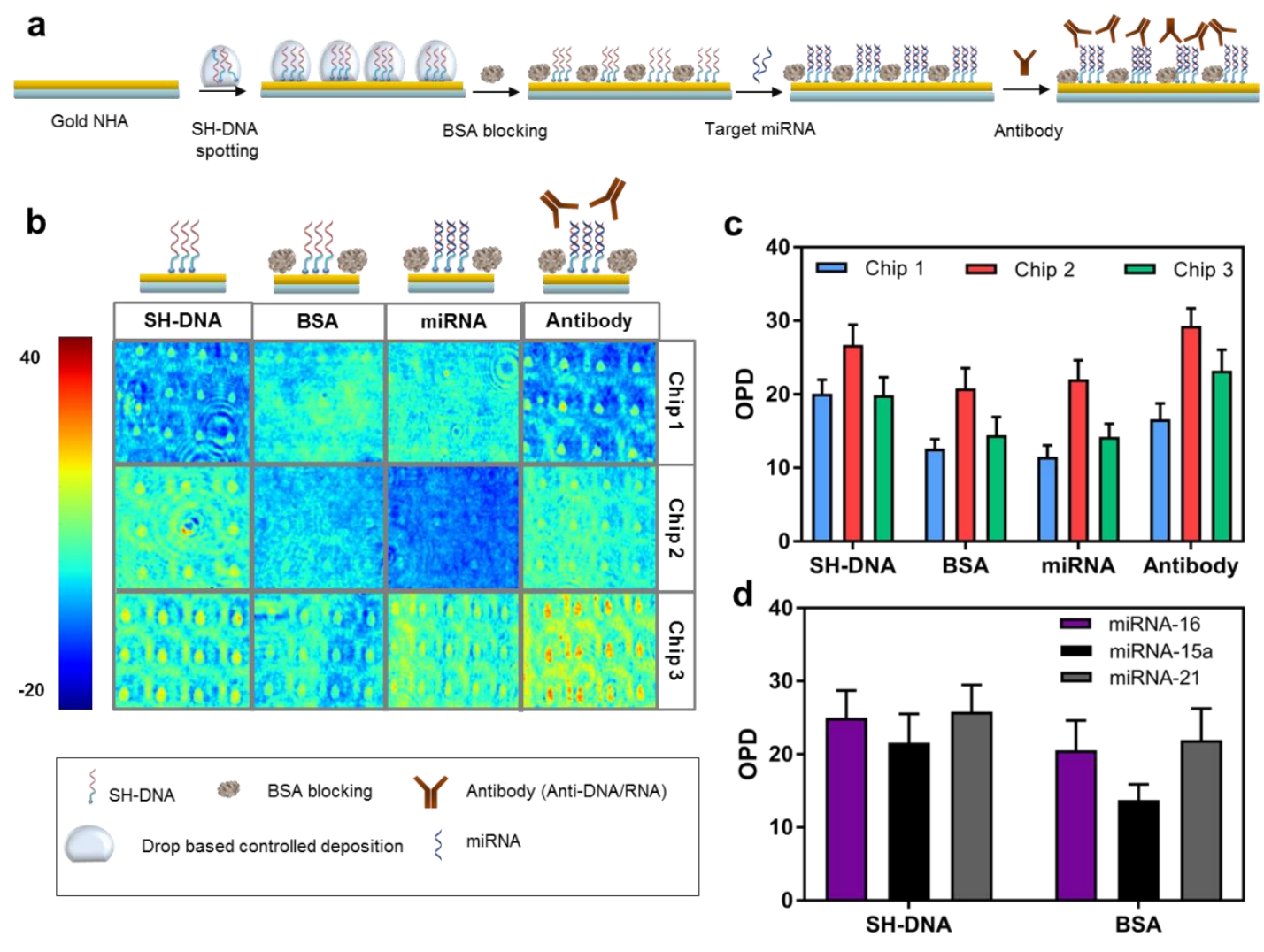

Figure 6: miRNA detection assay. a) Scheme of immobilization and detection of miRNA via hybridization assay with SH-DNA capture probes and antibody amplification. b) Intensity OPD maps of three microarrays in every step of the immobilization and detection protocol for miRNA (miRNA-16 $10 \mu \mathrm{M}$ ). c) Mean OPD \pm SD corresponding to images in figure 6b. d) Averaged OPD obtained for the immobilization of three different DNA capture probes (specific for miRNA-16, for miRNA-15a and for miRNA-21) in 20 chips. 
a

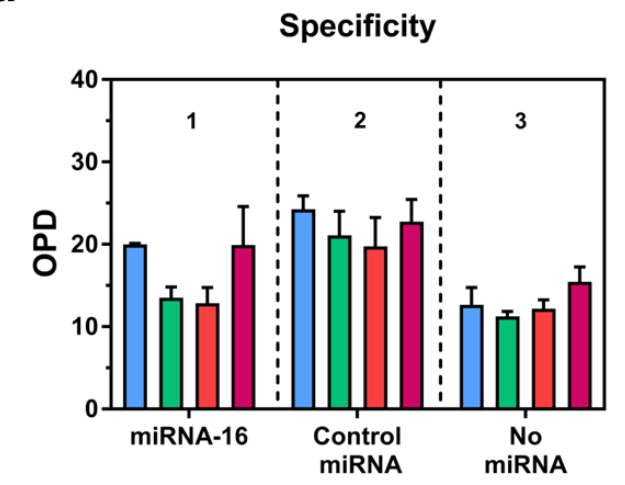

b

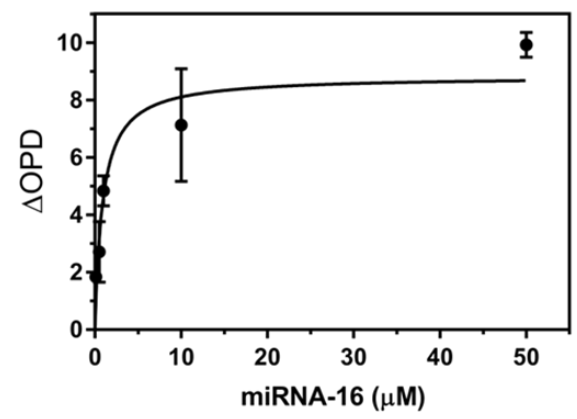

$\square$ SH-DNA $\square$ BSA miRNA $\square$ Antibody

Figure 7: a) Specificity study for miRNA-16 assay showing the variation in the OPD in 3 different conditions: (1) specific assay with target miRNA-16 $(10 \mu \mathrm{M})$ followed by anti-DNA/RNA; (2) addition of non-complementary miRNA (miRNA-21 = $10 \mu \mathrm{M}$ ) followed by anti-DNA/RNA; (3) no addition of any miRNA (i.e. only buffer) followed by anti-DNA/RNA. All data show mean OPD \pm SD of triplicate measurements. b) Calibration curve for miRNA-16 in PBS. The signals correspond to the amplification step performed with [anti-DNA/RNA] $=80 \mu \mathrm{gL}^{-1}$. All data show mean $\triangle \mathrm{OPD} \pm \mathrm{SD}$ of triplicate measurements. 